\title{
Entramar conocimientos y saberes necesarios para la educación del futuro (sobre las propuestas pedagógicas de Edgar Morin)
}

Link knowledge and knowledge necessary for the education of the future (on the pedagogical proposals of Edgar Morin)

\author{
Nelson Vallejo-Gómez \\ Secretario General de la Association pour la Pensée Complexe (París) \\ nvallejo@yahoo.fr
}

EDGAR MORIN Y EL PENSAMIENTO COMPLEJO
MONOGRÁFICO COORDINADO POR JOSÉ LUIS SOLANA RUIZ (Universidad de Jaén)

RESUMEN

La difusión de un pensamiento complejo, que Edgar Morin cree necesaria para enfrentar la situación de crisis global en la que se halla nuestro planeta, requiere una reforma de la educación escolar. Morin ha expuesto sus ideas sobre la reforma de la enseñanza en las jornadas temáticas que organizó y dirigió para responder a la consulta que Claude Allègre, por entonces ministro de educación, hizo sobre qué saberes enseñar en los institutos franceses, y en el libro "Los siete saberes necesarios para la educación del futuro", que le fue encargado por la UNESCO. En el presente texto, en primer lugar, se expone el sentido de esas jornadas y los temas, de naturaleza multidimensional e interdisciplinar, que se abordaron en ellas; y, en segundo lugar, se ofrece un testimonio personal del proceso de gestación, elaboración y difusión de Los siete saberes.

\section{ABSTRACT}

The diffusion of a complex knowledge, that Edgar Morin believes necessary to face the situation of global crisis in which in which is our planet, requires a reform of school education. Morin has presented his ideas on the reform of teaching in the thematic days he organized and directed to answer the question that Claude Allègre, at that time minister of education, made about what knowledge to teach in the French institutes, and in the book "Seven complex lessons in education for the future", which was commissioned by UNESCO. In the present text, first of all, the meaning of these conferences and the topics, of a multidimensional and interdisciplinary nature, that were discussed in them, and, secondly, a personal testimony of the process of gestation, elaboration and dissemination of Seven complex lessons in education for the future.

PALABRAS CLAVE

Edgar Morin | reforma de la educación | pensamiento complejo

KEYWORDS

Edgar Morin | education reform | complex knowledge

"Armés d'une ardente patience" (Arthur Rimbaud)

\section{Introducción}

Llegará el día en que los historiadores de la educación reconocerán en la obra de Edgar Morin un oporte mayor para entender lo que la ley francesa de orientación y programación para la refundación de la escuela republicana (Ley 2013-595) denomina como base común de conocimientos, competencias y cultura. (1)

Toda la obra de Morin es una propedeútica que converge hacia la emergencia de una base de ese tipo. Es una obra que contribuye a formar a la juventud y a mostrar que es posible responder a los grandes retos y desafíos que los conocimientos, las competencias y las culturas deben afrontar cuando se plantea la reforma del sistema escolar. Los Estados-nación han heredado de la Modernidad y del cientificismo decimonónico sistemas escolares cuyos métodos, lógicas y teorías educativas son difíciles de reformar. En efecto, la reforma a fondo de un sistema educativo cuestiona la pirámide de saberes y poderes, tanto como determinadas lógicas binarias que gobiernan la mentalidad de una cultura determinada.

Desde L'Homme et la mort (1951) hasta Enseigner à vivre. Manifeste pour changer l'éducation (2014), pasando por Le Paradigme perdu: la nature humaine (1973), L'Unité de l'Homme (1974), Relier les connaissances: le défi du XXI siècle (1999), La Tête bien faite (1999), Les sept savoirs nécessaires à l'éducation du futur (1999-2000), sin evocar aquí directamente, sino en filigrana, su opus magna, La 
Méthode (seis tomos, 1977-2004), Edgar Morin ha considerado fundamental una revolución cognitiva, que pasa por una reforma educativa, un cambio de paradigma y de mentalidad. Sin esa reforma, ¿cómo tomar consciencia de la necesaria metamorfosis de homo sapiens en humanidad?, metamorfosis necesaria, pues solo así parece plausible evitar que termine en catástrofe la aventura del ser humano en la Tierra.

El hombre, en su nivel de desarrollo actual, es capaz de ver y comprender numéricamente lo global; pero lo hace sin tomar consciencia de que es necesario pensar de manera global, es decir, de la necesidad de entramar (2) percepciones, sensaciones, sentimientos, ideas, pensamientos y teorías para afrontar los retos de la complejidad. "Nos encontramos en este mundo global confrontados a las dificultades del pensamiento global, que son las mismas que las del pensamiento complejo" (Morin 2015).

Sin la plena consciencia del pensar global, proseguirá la ceguera que ignora que el irrefrenado proceso de producción, consumo y destrucción del planeta es un círculo vicioso e infernal, ambicioso y egoísta, bárbaro y cruel, que conduce a la ruina individual y colectiva. La reforma de la educación requiere, también, de una ciencia con consciencia para humanizar el mundo y de una consciencia con ciencia para que la primera no se torne ideológica, fetichista ni egocéntrica.

\section{Entramar saberes para una reforma de las enseñanzas medias}

Fue necesaria una apuesta fallida de la derecha francesa para que Edgar Morin se convirtiese en "consejero oficial del Príncipe" y para que sus ideas, especialmente las relativas a la interdisciplinariedad, la transdisciplinariedad y al necesario entramado de conocimientos, competencias y culturas, fuesen tomadas en consideración -si bien transmutadas en argot administrativo- en los planes de los altos funcionarios encargados de la educación y la investigación, tanto en Francia como en los países de América Latina (países en los que la obra de Morin se encuentra ampliamente difundida y es comentada, aplicada, reconocida y apreciada).

Tras la disolución del parlamento francés por la derecha, la izquierda se instaló en Matignon (en mayo de 1997), y Claude Allègre, amigo personal y consejero especial de Lionel Jospin, ocupó un gran ministerio, el de Education Nacional, Investigación y Tecnología. Estaba acompañado en su equipo por Didier Dacunha-Castelle. Vivimos, entonces, la tercera cohabitación derecha-izquierda en el seno de la V República francesa: tras las de Mitterrand-Chirac (1986-1988) y Mitterrand-Balladur (1992-1995), la cohabitación Chirac-Jospin (1997-2002).

El ministro Claude Allègre deseaba emprender una reforma en profundidad de la enseñanza escolar, mediante una vasta consulta nacional, que respondiese a la siguiente cuestión: ¿Qué saberes enseñar en los institutos? Un sondeo rápido condujo a identificar al pedagogo del momento, al más presente y visible en los espacios públicos: Philippe Meirieu, director del INRP (Institut National de Recherche Pédagogique). Se le encargó la misión de conducir la referida consulta, poniendo a su disposición importantes recursos humanos y financieros. Su facundia y oportunismo político convirtieron rápidamente la consulta en una máquina mediática de ámbito nacional, que desbordó el marco pedagógico y la dimensión científica de la consulta. Fue por ello que el consejero especial D. Dacunha-Castelle decidió proponer al ministro que se pusiese al comité de consulta "bajo la jurisdicción" de un consejo científico y nombrar a Edgar Morin para ese consejo. Tuve el honor de ser el secretario general del referido consejo (entre noviembre de 1997 y octubre de 1998), como encargado de misión vinculado al gabinete del ministro.

En el libro Relier les connaissances. Le défi $d u X X^{e}$ siècle Morin relata ese nombramiento, y señala su dimension a la vez contradictoria y complementaria: "El ministro me había impuesto un consejo científico en el que pude introducir solo algunos nombres y al mismo tiempo él me había impuesto a ese consejo científico". Morin era consciente de que, independientemente de la calidad humana y personal de cada miembro, ese consejo no podía representar una "comunidad de sabios", pues no existe comunidad entre disciplinas muy diversas ni tampoco en el interior de disciplinas en las que se afrontan ideas opuestas. Igualmente, sabía que el carácter heteróclito del consejo y el tiempo limitado del que disponía para elaborar un informe no permitirían la emergencia de un consenso sobre algún "pensamiento común". Sabía, por último, que un comité de genios con ideas diversas "sólo puede llegar, en el mejor de los casos, a un promedio de sugestiones mediocres". Morin propuso llevar a cabo la tarea mediante unas "Jornadas temáticas" destinadas a "entramar los conocimientos", con un doble gran reto: 1) el de la globalidad (la inadecuación entre un saber fragmentado y unas realidades multidimensionales) y, por 
consiguiente, 2) la no-pertinencia de nuestro modo de conocimiento y enseñanza, que enseña a separar y no a entramar.

El propósito preliminar del presidente del consejo científico se encontró en seguida con la oposición de los poderosos egos, de los corporativismos disciplinares y de los sindicalistas. Una vez constituido el consejo por el ministro y que este se dedicó a otras obligaciones, las animosidades hacia un pensamiento complejo se transformaron en disimulados sarcasmos. Morin ha pintado con los siguientes términos sugestivos la primera reacción de los consejeros científicos: "Algunos querían, en su disciplina propia, llevar a cabo la revisión de los programas, otros preconizaban su modernización, otros su aligeramiento. El industrial del consejo [que se convirtió más tarde en ministro de economía en el primer gobierno de Jean-Pierre Raffarin] exigía que se pusiesen los pies sobre la tierra, es decir, que se pusiese la enseñanza al servicio de la empresa" (Morin 1999). Con su legendario humor y su arte para hacer razonar el sentido de las palabras dejando emerger la complejidad de los seres y de las situaciones, Morin concluyó el cuadro diciendo: "Como siempre, cada uno estaba seguro de estar situado en lo concreto, defendiendo su idea fija".

Durante varios días, Morin emprendió una amplia consulta. El consejero especial del ministro quería tener un documento que sirviese como marco o enfoque general. Le preparamos una serie de textos con el título Articular los saberes, que llevaban como portada la imagen de una famosa pintura de Giuseppe Arcimboldo, el Bibliotecario (1566): un triángulo de libros del que emerge una cabeza; imagen que relacionamos con un texto de Montaigne, referido a la mente (a "la cabeza"), que colocamos como exergo a la recopilación de textos: "Mejor bien constituida que bien llena" (una profundización de este planteamiento se encuentra en el libro de Morin La tête bien faite). Posteriormente, para tranquilizar al gabinete del ministro, preocupado por la materialización del proyecto, Morin propuso que el consejo tuviese como misión principal la organización de las "Jornadas temáticas", con el fin de poner a prueba la maquinaria echándola a andar. Se trataba, igualmente, de dejar claro, desde el punto de vista estratégico, que nadie estaba siendo engañado y que no se iba a sacar del sombrero una respuesta preconfigurada para una cuestión tan altamente compleja (la de qué saberes enseñar en los institutos).

Por otro lado, había estado claro, desde el principio, que el consejo no tendría funciones programáticas. No se trataba de rehacer los programas de enseñanza. Y, por lo demás, Morin pensaba que en lugar de "programa" se debería hablar en la educación nacional de "guías de orientación". Era necesario, pues, resisitir a la tendencia programática y al pragmatismo que veíamos emerger en la consulta, y privilegiar el aspecto reflexivo propuesto por el consejo científico. Ante el escepticismo y la estupefacción, Morin pareció desfallecer, pero el consejero especial zanjó y concluyó con alegría, en nombre del ministro, esa primera sesión del consejo: "Morin propone unas jornadas temáticas: ¡que se hagan! ¡Que cada uno haga sus propuestas!".

Para llevar a cabo las jornadas, Morin propuso que se trabajase mediante comisiones limitadas y temáticas, interdisciplinares, e invitó a los miembros del consejo a participar en ellas según sus afinidades electivas y su buena voluntad. Finalmente, el consejo en plenario solo se reunió (en la calle De Grenelle) dos veces: una para dar cuenta de la preparación de las jornadas y otra para presentar el proyecto del Informe Morin. ¿Qué saberes enseñar en los institutos?, informe elaborado a partir de las principales ideas propuestas en las jornadas. Entre las personalidades implicadas, las más predispuestas, asíduas y generosas durante todo el periodo del consejo fueron las siguientes: Alain Touraine, Yves Bonnefoy, Mireille Delmas-Marty, Marc Fumaroli, Pierre Léna, Philippe Meirieu y el Rector de la Academia de París, René Blanchet. Con el acuerdo de Morin, vinculamos a los preparativos de las jornadas y a la reflexión a tres profesores de instituto, que podían ilustrarnos de manera más detallada sobre cómo es la enseñanza escolar "sobre el terreno": Arnaud Guigue, Thomas Morvan y François L'Yvonnet.

La intuición de Edgar Morin, al proponer esas jornadas temáticas, era que hay "objetos", conceptos y teorías propias de cada disciplina científica, pero que hay igualmente "objetos" propios del conocimiento humano y del pensamiento complejo. El espíritu separa por necesidad de precisión y control, y entrama por estrategia de trayectoria, coherencia, globalidad y finalidad. Esos "objetos" deberían ser el esquema sustentador, el fundamento del entramado y de la articulación significante para una verdadera base. Los "objetos" disciplinares y su saber lógicamente fragmentado, su logística, deberían ser complementarios, mediante escalonamientos estratégicos, en el marco de un proyecto pedagógico integral, global e interdisciplinario.

A partir de textos sobre la educación, la ciencia y la cultura, la interdisciplinariedad, la transdisciplinariedad, el Emilio de Rousseau y la laicidad, Morin fue siempre consciente de que las 
enseñanzas proporcionadas a los alumnos durante el siglo XX, así como las maneras de enseñar, se inscribían aún en la lógica clásica del siglo pasado y no preparaban para los retos del siglo XXI (la era de lo numérico, la globalidad, la mundialización y la aceleración de todos los procesos); peor aún: no tenían en cuenta el cambio de paradigma y de mentalidades que imponen las ciencias contemporaneas.

En consecuencia, esas enseñanzas precisaban de una reforma integral del sistema educativo, de su organización, sus programas y su programación, su evaluación y sus objetivos. No se trataba de una enésima reforma administrativa o política, sino de una reforma del pensamiento, de una metamorfosis mental para pensar al actuar y obrar pensando, como preconizaba Henri Bergson.

Es cierto que reformar en complejidad, integralidad y globalidad es una enorme ambición, una empresa histórica, tanto a título individual como colectivo, pues esa reforma es ante todo ética, es decir, supone una manera de comportarse, de gobernar (para una "democracia cognitiva" y una "política de civilización"), de pensar y de vivir (para una "ética de entramado"); en suma, para Morin, lo que está en juego en la reforma de la educación es, nada menos, que "el porvenir de la humanidad". Pero, a pesar de esa ambiciosa finalidad, la reforma ha de comenzar en primer lugar por un trabajo consciente sobre nosotros mismos, para continuar cultivándonos y educándonos, y para permanecer libres y conscientes en la creación de nuestras ideas, nuestros hábitos y nuestras costumbres.

Si la cuestión antropológica permanece como uno de los envites de la educación, es forzoso constatar, con Le Paradigme perdu de Edgar Morin, que homo sapiens solo es aún un homínido, un primate mejorado en su naturaleza gracias a las emergencias culturales. Pero su humanidad, propiamente dicha, se encuentra aún en rodaje, pendiente de posibles metamorfosis y a la búsqueda de estas. De ahí la necesidad de una enseñanza que consagre unas nuevas humanidades, inspiradas en un método de pensamiento complejo, fortificadas con una ciencia consciente y con una consciencia científica. Son necesarias, en efecto, enseñanzas que sitúen al ser humano en el mundo y la sociedad, entramando ámbitos en tanto que sujeto múltiple, diverso y complejo. Los seres humanos solo existen cuando entraman y porque se encuentran en ciruítos naturales, mentales y sociales entramados, porque son en un "archipiélagos de relación", en palabras del poeta y pensador Édouard Glissant ("archipiélagos de entra-mar", diría yo). El hombre es naturaleza-individuo-sociedad o, si se prefiere, materia-espíritucultura. Y, además, puede trascenderse en ser histórico y en ejemplo para las generaciones futuras (sobre la dimensión antirreduccionista de la antropología compleja de Morin, véase Solana 1999).

La tesis de Edgar Morin para los trabajos del consejo era que el desarrollo de disciplinas científicas, desde las reformas universitarias del siglo XIX, inspirado en la taxonomía, el cientismo y el cartesianismo, divide y compartimenta cada vez más el campo del saber, conduciendo así a un conocimiento que no percibe la tríada naturaleza-individuo-sociedad, que, en definitiva, no permite ya que esos "objetos" emerjan y sean pensados en relación y en circuito interconectado. Ahora bien, es el mismo progreso científico (en física cuántica, biología molecular, cosmología, ecosistémica, antropología y prehistoria, ciencias cognitivas, ciencias de la vida y de la Tierra...) el que exige, desde comienzos del siglo XX, una reforma de la enseñanza escolar, de los métodos de su elaboración, concepción y aprendizaje.

El sentido de las jornadas temáticas era proponer, en primer lugar, elementos de información y reflexión para generar una cultura laica, con la esperanza de que esa cultura pudiese dotar intelectualmente a los alumnos de una base común, con el fin de que puedan aprender a situarse en el mundo y a asumir una condición humana multipolar, confeccionada mediante la suma e integración de distintas identidades (familiar, social, cultural, política, nacional, mundial). Además, se tenía la esperanza de que esa cultura también pudiese ayudar a los alumnos franceses a conocer y vivir los valores de la República francesa con una perspectiva multicultural, pues la multiculturalidad es parte y constituyente de la historia de Francia y de Europa, así como un valor indisociable de la historia de la condición humana en el planeta Tierra.

No cabe duda de que la cosmología contemporánea ha resucitado y renovado nuestro conocimiento del mundo. Las ciencias de la Tierra y la ecología científica han aportado un nuevo conocimeinto sobre nuestro planeta. Además, las nuevas ciencias biológicas, las ciencias cognitivas y los nuevos datos recabados en antropología y prehistoria han permitivo concebir la naturaleza, los individuos humanos y la sociedad en y más allá de la evolución viviente. Esas ciencias aportan una comprensión científica del misterio de la condición humana, en tanto que unidad múltiple y diversa (este aspecto de la antropología moriniana está especialmente bien tratado en Solana 1995). 
En el espíritu pedagógico de la complejidad es importante que el niño-alumno pueda reconocer su condición de ser humano, que es la de un individuo multipolar que depende de la relación naturalezacultura, que padece la muerte (como todo animal, pero disponiendo de una fuerza espiritual que hace de él el único ser vivo que cree en una vida más allá de la muerte), que posee una memoria individual y una historia colectiva, y que se construye como un sujeto consciente de ser uno y múltiple a la vez, en el que los contrarios pueden ser también complementarios.

Al proponer como marco o contexto de las cuatro primeras jornadas el Mundo, la Tierra, la Vida y la Humanidad, se trataba, para Edgar Morin, de plantear en conjunto unos "objetos" que favoreciesen la articulación de los saberes. Esos "objetos" pueden ser identificados por los niños-alumnos. En consecuencia, sería misión de los profesores conocer esos "objetos" y enseñar a los alumnos a conocerlos, a partir de un proyecto pedagógico interdisciplinar que permita plantear la condición humana en sus arraigamientos, sus determinaciones y sus problemas generales, individuales y colectivos.

Con esos "objetos", Morin proponía igualmente recuperar las grandes cuestiones que no han cesado de agitar la consciencia humana y que todo espíritu adolescente se plantea: ¿Qué somos? ¿De dónde venimos? ¿Hacia dónde vamos? Esas interrogaciones se encuentran alimentadas y renovadas de un modo más rico gracias a los diferentes aportes de las ciencias contemporáneas.

La cuarta jornada abordó la Humanidad en tanto que "objeto" de estudio. El proceso de hominización permanece en parte enigmático; un "eslabón perdido" aviva siempre la imaginación desde la Antigüedad. La Prehistoria, la Genética molecular, la Psicología y la Sociología muestran la importancia del desorden, del azar, del caos, del tercero excluido y de la eco-re-organización en la relación constituyente naturaleza-individuo-sociedad y en la relación significante cerebro-espíritu-cultura. Sea cual sea el origen, la confesión, el color de piel de un ser humano, esas relaciones subsisten y pueden ser constatadas en la emergencia de homo sapiens considerado como "objeto" de conocimiento fundamental. Los nuevos aportes científicos contribuyen a que se conozca mejor el proceso de formación de una consciencia humana, social y ética, de una Humanidad hecha $100 \%$ de cultura y $100 \%$ de naturaleza, y así su aprendizaje en términos de física, química, derecho, sociología, política, historia, lenguas, culturas y poesía sería una oportunidad para la paz y el bien vivir de los hombres sobre la Tierra.

Enseñar a los niños-alumnos a responder a las cuestiones fundamentales, a tomar consciencia de su condición humana y a saber situarse en el mundo local y global pasa también por vías distintas a las tradicionalmente consideradas como científicas; y de ahí la necesidad de una quinta jornada temática dedicada a las aportaciones de las lenguas, las civilizaciones, la literatura, las artes y el cine. Esos alimentos espirituales ayudan a captar las realidades humanas y a tomar consciencia de ellas; de manera más concreta, ayudan a aprehender las relaciones entre personas y sus elecciones afectivas, las subjetividades individuales, la inscripción en una familia, una tribu de pertenencia, una sociedad, una nación, una confesión, una historia; ayudan, en definitiva, a tomar consciencia de los caracteres complejos de la condición humana. Es por ello que Morin, con el bagaje de sus propias investigaciones sobre el cine, quiso que hubiese una jornada dedicada enteramente a esos "objetos" y pidió que no fuesen abordados de manera formal, sino como experiencias existenciales que conciernen a relatos y vivencias, así como a la identidad misma de los adolescentes (que es una identidad "en rodaje"), y como manifestaciones culturales que permiten experimentar el hecho de pensar por sí mismo y de asumirse como sujeto autónomo, libre y responsable.

Dedicada a la Historia, la sexta jornada temática estuvo en estrecha relación con la dedicada a la Humanidad. El hombre es el animal histórico por excelencia. Mediante la historia, una sociedad, una nación, construye sus referentes y traza su porvenir. La instrucción pública obligatoria tiene entre sus deberes el de procurar que cada niño-alumno pueda enraizarse en la historia y en la lengua-cultura de su país (francesa en el caso que nos ocupa), y que lo haga tomando consciencia de sus fuentes plurales, de su multiculturalidad, de su dimensión europea y mundial. Esa sexta jornada debía afrontar el problema clave de la interpretación histórica, de las memorias individuales y comunitarias, de las vinculaciones y dependencias contradictorias, para reafirmar el rol indispensable de la historia nacional, la laicidad y los valores de la República para formar la consciencia cívica, el vínculo público solidario y responsable de una sociedad constituida mediante instituciones republicanas y democráticas.

La séptima jornada estuvo dedicada al estudio de "las culturas adolescentes". La hipótesis de Morin era que, por varias razones, algunas quizás justificadas (otras seguro que no), la enseñanza clásica se efectúa en un escenario de confrontación entre dos mundos que se ignoran y que desconfían uno del otro: el mundo del adulto y el mundo del niño-adolescente. Ahora bien, los aportes de las ciencias 
(Psicología Social, ciencias cognitivas, Sociología de "las tribus urbanas"...) han mostrado que los mecanismos de aprendizaje de los niños, como el proceso mismo de aprender a lo largo de toda la vida, son complejos, y por eso necesitan métodos de enseñanza que se hagan cargo de esa complejidad, que la asuman. El profesor puede ser el mejor en su disciplina, conocer muy bien todos sus contenidos, pero puede fracasar en el proceso de enseñanza si ignora todo de sus alumnos y desconoce el contexto de la vida escolar en general.

Consciente de que no basta con enunciar la necesidad de entramar los conocimientos de manera compleja para encontrar los "objetos" articuladores de los entramados, Morin propuso que la última jornada estuviese dedicada a los principios, los conceptos y las herramientas intelectivas que pueden facilitar esos entramados; y también a métodos de conocimiento (racionalidad, cientificidad, medios no científicos de aprender) y a reflexionar sobre las relaciones -con frecuencia conflictuales- entre ciencia y filosofía, y sobre las relaciones entre las ciencias llamadas "humanas" (¿no "naturales"?) y las llamadas "naturales" (¿"inhumanas"?). Fue así como se proyectó la jornada "Entramar conocimientos".

Las jornadas temáticas apostaron por la resurrección concomitante de "objetos" y de grandes problemas, y en ellas se propuso que el profesorado pusiese su práctica y sus enseñanzas a tono con las grandes interrogaciones que todo ser humano y en particular todo adolescente se plantea, así como la necesidad de estimular la curiosidad por la tríada naturaleza-individuo-sociedad, tríada que es velada, perdida de vista, por "los objetos" disciplinares. Una de las misiones importantes de un enseñante del siglo XXI, volvió a recordarse, debe ser la de favorecer la capacidad mental para contextualizar y globalizar todo conocimiento, algo que las enseñanzas meramente disciplinares, por importantes e interesantes que sean, no permiten.

Por otra parte, las jornadas buscaron alentar al profesorado en una gigantesca y admirable tarea, que forma parte de los objetivos de la enseñanza y de los cometidos propios del profesorado: la de contribuir concretamente, en su práctica, a la elaboración de unas nuevas humanidades que permitan a cada uno reconocer lo humano en sus arraigamientos físicos, biológicos, sociales y culturales, y sobre todo en sus realizaciones espirituales; de unas nuevas humanidades que permitan reconocer lo humano y reconocer a los demás, a los otros, como seres humanos complejos, y que doten a las personas de la capacidad para situarse de manera entramada en su mundo, su tierra, su historia, su sociedad y su cultura.

Esas nuevas humanidades resultan también indispensables para la regeneración de una cultura humanista laica, pues una verdadera cultura humanista no es una imposición ideológica ni un etnocentrismo identitario, sino que forma parte de la aventura que supone la metamorfosis del hombre hacia la concreción y el logro de su condición de humanidad. Se trata de unas nuevas humanidades que han de estimular la aptitud de los seres humanos para la apertura, la problematización, la interrogación y el asombro, así como la consciencia y la voluntad de afrontar la era planetaria y la complejidad del mundo.

\section{Los siete saberes necesarios para la educación del futuro}

Lo que a continuación presento es un testimonio sobre el proceso organizativo y emergente que llevó a la idea, el contenido y la elaboración del texto Los siete saberes necesarios para la educación del futuro. Dar testimonio supone el haber vivido algo en carne propia, tener una vivencia memorable y las pruebas para compartirla. ¿En qué sentido la vivencia de la gestación y elaboración de Los siete saberes merece rememoración y ejemplaridad, es decir, transciende la simple acción individual, deja de ser un hecho personal y se convierte en referente colectivo? (3) Eso es lo que precisamente trataremos de mostrar contando el proceso de creación-emergencia de Los siete saberes.

Muchos congresos internacionales, sobre todo en América Latina, muestran que estamos en presencia de algo que compartimos muchas personas a través de la red mundial de la UNESCO, pero también más allá de dicha red. Se trata de algo que, con ya casi veinte años tras haber sido presentado en París en la Conferencia General de la UNESCO, permanece cada día como contemporáneo, es decir, como pertinente, como algo que no sufre de caducidad ni de esa condición tan de moda en el mundo de la cultura globalizada: la condición de desechable.

Me estoy refiriendo al texto Los siete saberes necesarios para la educación del futuro, publicado en octubre de 1999 por iniciativa de la dirección del programa mundial de la Organización de las Naciones Unidas "Educación para un futuro sostenible" (cuyo director durante todo el período requerido para su 
implementación fue Gustavo López Ospina), una obra que pone en escena una propuesta de educación para la humanidad en la era planetaria.

¿Cómo nació la idea de Los siete saberes? ¿Cómo se elaboró el texto?, ¿con qué receta o método?, ¿con qué condimentos conceptuales?, ¿en qué cocina y en qué condiciones? ¿Quiénes fueron los actores clave en el proceso de elaboración de ese texto de referencia? ¿Cómo fue presentado en París? ¿Cómo fue acogido por la comunidad educativa internacional? ¿Cuál ha sido su suerte desde entonces? ¿Por qué se ha convertido con el tiempo en un texto a su vez clásico y moderno cuando se habla de educación para la era planetaria? ¿Qué hay, pues, tan especial en y con el texto Los siete saberes necesarios para la educación del futuro? El hecho de haber sido uno de los actores en tan extraordinaria aventura del pensamiento me permite poder responder a esas preguntas, en particular a la pregunta por el origen o el nacimiento de la idea, y poder contar cómo fue el proceso interactivo de elaboración, organización y emergencia de ese texto.

Creo que puedo decir, sin temor, pero con cierto temblor y respeto, que el destino me hizo partícipe de la maravillosa aventura que supuso la concepción de la propuesta, la organización del proceso, la química de las ideas, la pelea política por guardar la libertad de pensamiento, la redacción aparentemente contradictoria, el ir y venir, desde y con la obra de Edgar Morin, animando un dispositivo de auto-eco-reorganización del texto mismo. En suma, como en toda creación humana, íbamos quitando y poniendo materia conceptual a lo que solamente era en un principio una propuesta-base de 25 páginas, que tenía por título provisional Para la educación del siglo XXI; es decir, íbamos reflexionando a su vez sobre su contenido y su forma, con el fin de consolidar un texto final, que no excediera unas 70 páginas, que tuviera suficiente fuerza propia para ser a su vez una propuesta abierta, crítica y autocrítica, que fuese incitativo sin ser vago, que fuese ambicioso sin ser excesivo, que pudiese ser leído por un individuo medianamente ilustrado. Un texto que, además, integrase el paradigma científico occidental, pero librándolo, mediante la reflexión y la crítica, de sus proyecciones como ideología del poder ejercido por Occidente; que fuese sensible a la docta ignorancia y al diálogo del alma consigo misma (definición del pensamiento según Aristóteles); que fuese a la vez poético y prosaico. Un texto, en fin, que fuese de veras un acicate para una reforma educativa de carácter más paradigmático que programático, y que pudiese incitar a los responsables políticos y a los educadores a concebir una educación capaz de promover una inteligencia general, apta para referirse a lo complejo de manera multidimensional, en un contexto dado y una concepción global.

En el prefacio publicado en el texto oficial y firmado por el director general de la UNESCO, Federico Mayor, cuando este da las gracias a la institución por el trabajo realizado y se refiere al desafío que representa la reforma educativa como fundamento para poder reformar el pensamiento de las nuevas generaciones, consta lo siguiente: "Mis más especiales agradecimientos van para Edgar Morin por haber aceptado estimular, junto con la UNESCO, una reflexión que facilite dicho debate [el debate sobre la reforma de la educación] en el marco del proyecto transdisciplinario Educación para un futuro sostenible. Expreso igualmente todos mis agradecimientos a los expertos internacionales que han contribuido a enriquecer este texto con sus sugerencias y muy especialmente a Nelson Vallejo-Gómez".

En la edición original de Los siete saberes, Edgar Morin asume la autoría general del texto, precisando, sin embargo, los aportes de otras personas y sus agradecimientos a estos en los siguientes términos: "este texto ha sido sometido a la consideración de personalidades universitarias y de funcionarios del Este y del Oeste, del Norte y del Sur, entre los cuales están [sigue una lista de una veintena de expertos consultados por la UNESCO]". En el párrafo siguiente, Morin aclara una autoría suplementaria en términos que fueron negociados de la siguiente manera: "Nelson Vallejo-Gómez fue el encargado por la UNESCO de retomar e integrar las propuestas y de formular sus propios aportes. El texto resultante contó con mi aprobación". Esa negociación forma parte de la "historia secreta" de la elaboración de Los siete saberes, pues en el contrato original estaba previsto el reconocimiento tradicional de coautoría, lo que en ese tipo de trabajos se precisa con la formulación: "en colaboración con fulano o mengano". Esos aportes fueron sin duda menores, no necesariamente en cantidad o en calidad, sino simplemente cuando se los compara con la monumental obra de Edgar Morin.

Sin embargo, dichos aportes y el trabajo mismo efectuado por -en palabras de Morin- "el encargado por la UNESCO de retomar e integrar las propuestas y de formular sus propios aportes", que son el resultado de lo que se puede llamar el rol de colaborador del texto, permitieron echar a andar el proyecto y el texto (como reza el verso de Antonio Machado, que tanto le gusta citar a Morin: "Caminante no hay camino, se hace camino al andar"). 
El texto original fue elaborado en francés y, en su momento, se presentó también adicionalmente en inglés y en castellano, traducido por la profesora Mercedes Vallejo, de la Universidad Pontificia Bolivariana de Medellín (Colombia), con el apoyo atento, para mejorar la legibilidad y corregirlo gramaticalmente en los tres idiomas, de Françoise Girard, asistente de la dirección del programa Educación para un futuro sostenible.

Diez años después, nos encontramos ante un documento que tiene vida propia, que se ha convertido en un faro mundial en el tema educativo, que ha sido traducido a treinta y dos idiomas, y que circula hoy libremente por miles de redes. Los siete saberes quizás sea el primer texto que trata de manera fundamental (entretejiendo el destino de la especie, del individuo, de la sociedad, de la historia y del planeta Tierra) el tema de la educación para el nuevo hombre del siglo XXI, para el hombre de la era planetaria.

He visto cómo ese texto ha sido leído y discutido en universidades de París, así como en escuelas de localidades de los Andes peruanos a las que se llega todavía a caballo. He visto, también, cómo cada uno de sus siete capítulos han sido estudiados por grupos de profesores de diferentes regiones de Colombia durante seis meses, para luego presentar sus comentarios en un encuentro nacional organizado en Bogotá por Marco Antonio Velilla Moreno y la Corporación Complexus para el Desarrollo, en noviembre de 2000, en presencia de Edgar Morin y del Ministro de Educación de Colombia, como contribución a la reforma educativa en ese país. Los siete saberes es constantemente "sometido a escuchas pedagógicas" por docentes, alumnos y expertos de diferentes disciplinas, como ocurrió en el congreso conmemorativo de los diez años de la obra que nos ocupa, celebrado en Fortaleza (Brasil) en 2010. También ha sido y sigue siendo sometido a "escuchas" por parte de empresarios y políticos. Eso se debe a que estamos en presencia de un texto abierto que permite justamente establecer un dialogo constructivo entre su contenido, su forma y los diversos contextos culturales en que es leído, discutido y sometido a crítica.

No me parece fortuito, producto del azar, que el referido congreso conmemorativo se realizara en Brasil, pues se trata de un país joven y dinámico que está a la vanguardia de una modernidad abierta, innovadora, creativa, de un país que ha sido en América Latina pionero en temáticas relativas al pensamiento complejo. Recordemos que fue justamente en Brasil, en Río de Janeiro, en la Universidad Candido Mendes, donde la Association pour la Pensée Complexe (APC) organizó, con el apoyo de la UNESCO y de otras instituciones afiliadas, el Primer Congreso Inter Latino de Pensamiento Complejo (CILPEC), del 8 al 11 de septiembre de 1998. Hay que subrayar que, con motivo del CILPEC, la APC logró también algo que nunca antes se había hecho en Brasil, ni tampoco en América Latina: que días antes de la inauguración del congreso, el 7 de septiembre, un gran periódico, el Jornal do Brasil, publicase un suplemento especial, de ocho páginas, enteramente dedicado a la presentación de dicho congreso, a sus temáticas, junto con una larga entrevista a Edgar Morin. En esa entrevista, Morin explicaba por qué considera a Brasil en particular y a América Latina en general como el caldo de cultivo más fértil para la experimentación de la reforma del pensamiento y esbozaba ya lo que, desde entonces, ha tomado fuerza y consistencia bajo la denominación de "un pensamiento del Sur" (4). Tuve el honor de concebir y realizar dicho suplemento, con el apoyo del entonces cónsul general de Francia en Río de Janeiro, Denis Delbourg.

Sucede con los textos clásicos, como también con los grandes descubrimientos, algo curioso: con el tiempo, se diluyen el contexto y las particularidades, lo prosaico y lo trivial, las pasiones, las penas y las alegrías en que fueron elaborados o descubiertos. Sucede, también, que esos textos y esos descubrimientos se han hecho ya universales, alcanzando el estatuto de patrimonio inmaterial de la humanidad y beneficiando a toda las personas, sin excepción de raza, cultura o condición social. De ese modo, su autor o autores, los actores y la historia que los engendró, quedan relegados a lo que podríamos llamar como "hechos menores". No es que desaparezcan, pues basta con repensar, revivir, el cuerpo de todo gran texto para saber y sentir que detrás de lo universal, de cada gran hombre o de cada gran idea, hay personas comunes y corrientes, hay un contexto histórico especifico, hay destinos que combinan lo individual, lo social, lo histórico y lo cultural; y, ante todo, hay un impulso que trasciende lo local y aspira a lo global. Pues bien: así sucede con Los siete saberes. Recordemos que, en el comienzo, el título del texto no era ese, sino Reflexiones para la educación del mañana. Sin embargo, mientras vivamos y contemos con fuerzas, podremos dar testimonio de la gestación de la obra y recordar el contexto, los actores y el método o camino que se recorrió para que fuese un hito en la educación para la era planetaria.

Fue en el marco de la $30^{\mathrm{a}}$ sesión de la conferencia general de la UNESCO, el 27 de octubre de 1999, que el director general, Federico Mayor, presentó oficialmente, con Edgar Morin, Gustavo López Ospina y 
mi persona, el original en francés de Los siete saberes. Para los mandarines de la educación tradicional, dicho texto parecía algo marginal; se extrañaban de la importancia, el reconocimiento y el protocolo que le concedía el director general de la UNESCO. Así mismo, al ver el sello de la UNESCO en un texto firmado por un solo pensador y que ese texto, en virtud de ese sello, accedía al rango de documento internacional como propuesta para la reforma educativa de los Estados miembros de la ONU, muchos burócratas de la red mundial de la ONU se sintieron despojados de sus controles de identidad disciplinaria. Era evidente, como constatamos con el tiempo y como los actores presentes presentían, que estábamos ante un texto histórico, es decir, frente a una magistral desviación en las propuestas sobre educación.

Los siete saberes tienen una historia propia y singular, en la que la pasión fogosa, el eros inspirador y la amistad reparadora fueron elementos decisivos para llevar a cabo un texto que, para muchos, en particular para el ala conservadora de la educación (que en la UNESCO tiene sus mandarines propios), resulta una propuesta abrumadora. El texto no ignora que enfrenta importantes desafíos. Pero los conservadores lo acusaron de inmediato de ausencia programática. Manifestaban, así, en realidad, el juego de poder y de concesiones entre tecnócratas disciplinarios, representantes de sindicatos y ONG mediante el cual se consensuan los "documentos oficiales" elaborados por los organismos intergubernamentales internacionales, documentos en los que lo que finalmente prima es un lenguaje apático y políticamente correcto.

Con todo, la primera propuesta para la realización del texto que nos ocupa se discutió en una reunión de trabajo, el 13 de febrero de 1998, que tuvo lugar en la sede de la "Misión Morin" para la reforma del liceo francés, en una de las dependencias del entonces Ministerio de Educación, Enseñanza Superior, Investigación y Tecnología (rue Descartes de París) (5), cuyo ministro era Claude Allègre. En esa reunión estuvieron presentes Edgar Morin, presidente de la APC, Gustavo López Ospina, director del programa transdisciplinario de la UNESCO Educación para un futuro sostenible, y yo como secretario general de la APC.

En esa primera reunión, simplemente se acordó la posibilidad de establecer un convenio entre la UNESCO y la APC, con el fin de elaborar una propuesta sobre educación a partir de los conceptos clave del pensamiento complejo, la obra de Morin, las experiencias adquiridas en las jornadas temáticas Entramar los conocimientos y las que pudiesen obtenerse de los talleres que debían de ealizarse durante el CILPEC. Deseoso de conocer de antemano cuál sería el margen de autonomía de la APC en la elaboración de un documento que tendría el sello de la UNESCO, Edgar Morin propuso que se organizara también una reunión con el director general, Federico Mayor. Yo, como secretariado general de la APC, fui el encargado de redactar un proyecto de convenio en el cual se estipulara como anexo el objetivo general del acuerdo, los objetivos específicos y la metodología de trabajo para la redacción del texto.

Responsable desde mediados de la década de 1990 de aportar a la UNESCO los instrumentos conceptuales para repensar nociones modernas que se han tornado inoperantes ("progreso", "desarrollo", "sostenibilidad"), en el marco del programa internacional lanzado por la Comisión para el Desarrollo Sostenible de la ONU sobre la Educación, la sensibilización del público y la formación para la viabilidad, Gustavo López Ospina se había acercado ya a la obra de Morin, en particular a sus libros Para salir del siglo XX (1981), Ciencia con consciencia (1982) y Tierra-Patria (1993). López Ospina sabía de entrada que no es posible una reforma política del modelo de desarrollo socioeconómico, que pudiese lograr implementar un desarrollo integral y multidimensional sostenible y sustentable, además de justo y solidario, sin una reforma educativa asumida como finalidad política fundamental por los responsables políticos y administrativos, por la sociedad civil y, sobre todo, por el profesorado. Como Morin señala, citando a los reformadores socialistas rusos del siglo XIX, la pregunta fundamental en toda reforma educativa es “¿Quién va a formar a los formadores?”, es decir: “¿Quién va a educar a los educadores?" Uno presiente que esa cuestión puede ser tan general como inabordable, pues ante ella lo primero que viene a una mentalidad racionalista clásica es la figura del "circulo vicioso" o la historia "del huevo y la gallina". Percibimos aquí las paradojas planteadas ya en la antigua Grecia por el presocrático Zenón de Elea, relativas a la liebre y la tortuga o a la división infinitesimal de la distancia entre cero y uno, basadas en una confusión entre el espacio real y la abstracción aritmética de este. También sabemos ya que el problema central del conocimiento del conocimiento no reside solo en la verdad o falsedad de las ideas, sino además en su dimensión paradigmática, es decir, en la manera como se organizan las ideas y los conocimientos sobre las distintas realidades (la cultura, la sociedad, el individuo, la naturaleza...). Por otro lado, la cuestión reside también tanto en el sujeto formador como en el objeto de la formación y, sobre todo, en la política de formación -que Morin denomina como "política del hombre o "política de 
civilización"-, es decir, en la propuesta organizadora, paradigmática, que una política educativa es capaz de asumir.

Retomando la pregunta de los reformadores socialistas rusos, uno podría responder que es "la política educativa" la encargada de formar a los formadores, de educar a los educadores. Pero todavía es necesario elaborar e implementar una política educativa que asuma de manera integral la reforma del pensamiento en la era planetaria. Esa política está esbozada en algunos planteamientos innovadores de distinto ámbito (local, nacional e internacional), pero no prima porque los intereses financieros y las identidades culturales de los Estados-nación mantienen todavía el tema educativo como un instrumento soberano para la reproducción de élites con idiosincrasia local.

La educación vehicula una promesa de universalidad que permanece siempre sin cumplirse, pues, de lo contrario, el individuo ilustrado accedería a la condición de identidad global, humana y planetaria. $Y$ esa condición es difícil de asumir porque implica la conciencia de un arraigamiento-desarraigado y un desarraigamiento-arraigado, en la cual se perfilan una sabiduría y una capacidad mental para estar fuera y dentro de la sociedad, fuera y dentro de la naturaleza, fuera y dentro del universo, es decir, para estar fuera y dentro de la parte y del todo. En términos cognitivos, es lo que se define, desde Sócrates hasta Edgar Morin, pasando por Nicolás de Cusa, como estar en y tener la condición de docta ignorancia.

El 21 de julio de 1998, Gustavo López Ospina organizó un almuerzo de trabajo en el último piso del edificio de la UNESCO en París, Place de Fontenoy, con la presencia de Federico Mayor, de Edgar Morin y de mi persona. En ese encuentro se estableció la metodología y las condiciones de elaboración del texto, se acordaron los términos del convenio entre la APC y la dirección del programa Educación para un futuro sostenible, y se hizo un pacto de caballeros entre el director general de la UNESCO y el presidente de la APC para asegurar total libertad de pensamiento en la formulación de la matriz generadora del texto. La redacción de base correría por cuenta de Edgar Morin, quien, por su parte, aceptó que dicha matriz o documento base fuese sometido a revisión y crítica por expertos en diferentes contextos culturales, así como que se pudiesen integrar en su texto algunas de las propuestas de esos expertos y los posibles aportes del secretario general de la APC, encargado por la UNESCO de integrar y fijar, bajo la mirada de Morin, el texto final. Se acordó que el objetivo específico del texto final fuese una contribución al debate internacional sobre la forma de reorientar la educación hacia el desarrollo sostenible. En el prólogo de la publicación original del texto, el director general de la UNESCO precisó dicho objetivo en estos términos: "Mi intención es que estas ideas [las que recoge Los siete saberes] susciten un debate que contribuya a ayudar a educadores y dirigentes a aclarar su propio pensamiento sobre este problema vital [el de una educación para el desarrollo sostenible]".

Detengámonos un momento en el diagnóstico, el contexto y la metodología que sostienen la elaboración de Los siete saberes. La humanidad ha logrado en los tres últimos siglos, desde el advenimiento de la Modernidad, y en particular durante la segunda mitad del siglo XX, un desarrollo enorme de las ciencias y la tecnología, que conlleva una hiperespecialización de las disciplinas que ha dado lugar a la desaparición del hombre, la sociedad y el mundo como "objetos" de conocimiento. De modo diferente, la obra de Morin platea la necesidad de repensar el individuo, la sociedad y la especie humana desde una visión global y una concepción multidimensional, entramando los conocimientos para pensar desde la complejidad. La educación debe promover una inteligencia general y global, contextualizada y compleja, que religue el hombre, la cultura y el mundo.

Al analizar lo ocurrido durante el siglo XX, Morin dice que ese siglo es una "era de hierro planetaria", es decir, una fase en el proceso de humanización. En esa línea, plantea que, si se quiere que durante el siglo XXI se avance en la constitución de la especie humana como humanidad, es necesario un nuevo humanismo. La humanidad deberá ser repensada con un nuevo paradigma, a partir de la tríada individuo $\leftrightarrow$ sociedad $\leftrightarrow$ especie. La especie humana es ya hoy una comunidad de destino planetario, habita un único territorio posible para su supervivencia, la Tierra-Patria, y su conversión en plena humanidad ha de realizarse mediante una antropoética.

Esa nueva era de la humanidad, la era planetaria, requiere de una educación que sea capaz de transmitir un conjunto de conocimientos fundamentales y, a la vez, de asumir los misterios y las incertidumbres que el avance y el incremento del saber generan; de una educación que, además, evite caer en tres tipos de trampas: la de la moda, en la que los conocimientos realmente fundamentales son sustituidos por simulacros pasajeros; la de una "diversidad cultural" que diluye y desintegra las identidades individuales y locales en el discurso oportunista del consensualismo; y la de una globalización que ofrece una visión unidimensional de las realidades socioculturales. 
Una visión maniquea podría presentar a Los siete saberes y al pensamiento complejo como la saga de un combate entre el "paradigma de disyunción" y el "paradigma de complejidad", cuando se trata, en realidad, como el mismo texto precisa, de "comprender un pensamiento que separa y que reduce junto con un pensamiento que distingue y que religa. No se trata de abandonar el conocimiento de las partes por el conocimiento de las totalidades ni el análisis por la síntesis; hay que conjugarlos". Se trata, pues, de "conjugar" (un verbo que utilizamos a menudo sin ser consciente de todo lo que significa). Por lo demás, Morin especifica en muchas partes de sus obra que el pensamiento complejo no es, como tal, un bálsamo o remedio mágico que asegure el buen pensar. La realidad es que el mismo pensamiento complejo ha de enfrentar sus propios desafíos.

\section{A modo de conclusión}

Según dice el refrán, "Nadie es profeta en su tierra". Las ideas de Edgar Morin sobre la educación han tenido, en especial a través de Los siete saberes, una amplia difusión en América Latina, pero no en Francia, donde apenas se le prestó atención al "informe Morin 1998" sobre la reforma de la educación secundaria (6). El ministro comendatario se encontró rápidamente desbordado por un sindicalismo de izquierdas refractario a cualquier reforma y por la incapacidad de innovación de una administración paquidérmica. Edgar Morin no se empeñó en que su informe fuese considerado. Hicimos nuestro trabajo, hasta donde nos dejaron, y partimos "armados de una ardiente paciencia" a la búsqueda y espera de nuevas oportunidades, que terminaron por llegar con el proyecto de Los siete saberes auspiciado por la UNESCO.

Edgar Morin puede ser considerado como el educador-tipo de una civilización terrestre en la era planetaria, como antaño, mutatis mutandis, Homero lo fue para la civilización de la antigüedad griega. Toda la obra moriniana converge en una especie de ética del bienpensar, en una base común para una política de civilización. Así pues, Edgar Morin jel educador planetario!

Morin ha sido siempre un rebelde para la institución universitaria escletotizada. Algunos se sorprenden de su celebridad tardía, ahora que su obra colosal se encuentra traducida a más de treinta lenguas (7), que ha recibido más de treinta doctorados Honoris causa en distintos países del mundo, y que se puede decir de Morin, con admiración por la obra y respeto por el hombre, lo que Alain Touraine dijo en 2001, cuando celebramos los 80 años de Edgar en la UNESCO: “¡He aquí un sociólogo planetario!" A lo que podemos añadir, retomando el título del homenaje que le rendimos entonces, organizado con la ayuda de Gustavo López Ospina y en presencia de Jack Lang y de Federico Mayor: "Morin, el humanista planetario".

\section{Notas}

1. Esa ley establece, en su artículo 13 modificado, que "la escolaridad obligatoria debe garantizar a cada alumno los medios necesarios para la adquisición de una base común de conocimientos, competencias y cultura, a la que contribuye el conjunto de enseñanzas dispensadas durante la escolaridad. Esa base común debe permitir la continuidad de los estudios y la construcción de un futuro personal y profesional, y debe preparar para el ejercicio de la ciudadanía".

2. Prefiero traducir así al español, como entramar en lugar de como religar, el término francés relier utilizado por Morin.

3. Con el tiempo, lo vivido por uno o varios individuos puede transformarse en una leyenda o bien, compartido por muchos, se convierte entonces en un hecho histórico relevante, en algo que marca un antes y un después para una comunidad. En este último caso, uno se refiere a lo vivido no ya como testimonio, sino como momento específico suficientemente cargado de significado como para ser punto temporal de referencia en un proceso de explicación y conocimiento. Los hechos históricos tienen propiamente que ver con la cultura, con la manera de medir el tiempo de los seres humanos en sociedad. Pero también tienen que ver con la vida individual de cada cual; sin el aporte singular de los individuos, no hay hecho histórico.

4. En la entrevista publicada por el periódico Jornal do Brasil, el 7 de septiembre de 1998, preguntaba a Morin lo siguiente: "Volviendo a la atención sostenida que encuentra su pensamiento en América Latina, ¿piensa usted que podrá salir de los países latinos un ejemplo de reflexión sobre la complejidad de la 
cual la vieja Europa pudiera nutrirse, y, en ese sentido, que este Primer Congreso Inter Latino para el Pensamiento Complejo pueda inspirar otros?" La respuesta de Morin fue la siguiente: "Los países latinos son caldos de culturas vivas que abren un espacio de esperanza y de porvenir para la vieja Europa. Hay, también, una gran vitalidad cultural, que comienza a tomar conciencia de ella misma. Hasta una época reciente, los países de América Latina se daban las espaldas los unos a los otros. En literatura, por ejemplo, los escritores de esos países solo empezaron a ser conocidos y reconocidos cuando fueron traducidos en París. En política, fue preciso un día retomar las palabras de Bolivar para encontrar otra unión que la meramente económica; la idea de unión, de una confederación latino-americana, hace su camino, ya que esos países tienen orígenes y lenguas comunes. Los países de América Latina viven, cada uno a su manera, las tragedias y riquezas de la complejidad. La riqueza de la complejidad es la unión de la diversidad y la unidad, las bellezas creadoras de los encuentros y de los mestizajes en todos los dominios. La tragedia de la complejidad es la amplitud de los antagonismos, que pueden ser destructivos. Como usted sabe, la democracia es un régimen complejo que se nutre de antagonismos de ideas, de intereses, pero que es capaz de regularlos pacíficamente a través de sus debates y de hacerlos productivos. En América Latina, el gran problema actual es que los antagonismos que la despedazan puedan volverse productivos. Así, los grandes antagonismos planetarios entre el Norte y el Sur, el Este y el Oeste, ricos y pobres, hiperdesarrollo y subdesarrollo están presentes y activos en América Latina. Algunos países incluso, como Colombia, viven a la temperatura de su propia destrucción. Pero sabemos que las fuerzas de vida y de creación pueden utilizar los procesos de destrucción para alimentar los procesos de regeneración. Por mi parte, yo creo, espero, que una nueva conciencia latinoamericana emergerá de esa situación única, que ella extraerá de la experiencia del Sur, de la técnica del Norte y del aporte de las culturas europeas y de las sabidurías asiáticas los recursos de un nuevo impulso creador, un mensaje de renacimiento cultural e intelectual, que podría aportar al mundo, como lo hicieron en los siglos XV y XVI las ciudades de la Toscana, que produjeron el Renacimiento europeo. Espero que el congreso verá la afirmación de un pensamiento meridional, capaz de universalidad, como lo fueron antaño los pensamientos mediterráneos de Atenas y de Roma, un pensamiento abierto capaz de integrar todos los aportes exteriores, pero que sepa que la finalidad de su esfuerzo histórico, así como la medida de lo humano, están no en eso que es cuantitativo sino en la calidad, en la calidad de la vida. El pensamiento meridional debe ser capaz de integrar en una racionalidad más abierta -racionalidad compleja justamente- la racionalidad tecno-cuantitativa del Norte. Esa racionalidad debe ser mensajera de las necesidades y aspiraciones de los oprimidos y los miserables, sin olvidar las minorías indias amenazadas de exterminio o desintegración cultural. Son las voces de la sensibilidad humana, del sufrimiento humano, de las desgracias humanas las que esa racionalidad debe ser capaz de hacer escuchar en lugar de ahogarlas. Ha de ser un pensamiento juvenil, que contenga el ardor y la revuelta contra el mal, y al mismo tiempo un pensamiento adulto, que no prometa ningún paraíso en la Tierra. Ha de trabajar por un mundo mejor, pero sabiendo que es una ilusión creer en el mejor de los mundos".

5. Tiene algo de irónico que la propuesta de elaborar un texto que podríamos llamar el "Manifiesto del pensamiento complejo" o "Propuesta para pensar el conocimiento desde el paradigma de la complejidad" haya nacido en la calle del filósofo emblemático del "gran paradigma de Occidente". Según señala Edgar Morin en varias partes de su obra, ese "gran paradigma de Occidente", basado en una concepción de la realidad (los individuos, la sociedad, la naturaleza...) dibujada mediante disyunciones y dicotomías (sujeto/objeto, alma/cuerpo, espíritu/materia, calidad/cantidad, finalidad/causalidad, sentimiento/razón, libertad/determinismo, existencia/esencia, entre otras), fue formulado por René Descartes.

6. Toda la vida y la obra de Edgar Morin han sido, particularmente en Francia, una consciente y profunda preparación para eso que podemos ilustrar mediante la famosa sentencia "predicar en el desierto", aunque la imagen pueda estar mal elegida para quien rehúsa ver el pensamiento complejo como una camarilla o un clan y sus funciones como las de un mesías (Morin 1989).

7. Cuando, en la década de 1970, los norteamericanos se interesaron por la obra de Morin y quisieron traducirla y difundirla en los Estados Unidos, un sociólogo del Collège de France, que hacía y deshacía cual si de un amo se tratase en el medio sociológico de la época, se opuso a ello alegando que, antes que a Morin, era necesario traducir a los "verdaderos sociólogos". Morin se lo tomó con humor y consideró el hecho como una bourde-dieu (de bourde: sandez y dieu: dios).

\section{Bibliografía}

Morin, Edgar

1989 “Mesías, de ningún modo", Gazeta de Antropología, nº 28 (3), 2012. 
1999a Relier les connaissances. Le défi du XXIe siècle. París, Éditions du Seuil.

1999b La tète bien faite. París, Éditions du Seuil.

1999c Los siete saberes necesarios para la educación del futuro. París, UNESCO/Santillana (trad. de Mercedes Vallejo-Gómez).

2015 Penser global. L'humain et son univers. París, Éditions FMSH y Éd. Robert Laffont.

Solana, José Luis

1995 "La unidad y diversidad del hombre en la antropología compleja de Edgar Morin", Gazeta de Antropología, $\mathrm{n}^{\circ}$ 11, artículo 02, http://hdl.handle.net/10481/13607.

1999 "Reduccionismos antropológicos y antropología compleja", Gazeta de Antropología, n 15, artículo 08, http://hdl.handle.net/10481/7531. 